\title{
Improving measurement 1: Harnessing the PROMise of outcome measures
}

Thomas D Dobbs ${ }^{1}$, Jeremy Rodrigues ${ }^{2}$, Andrew M Hart ${ }^{3}$, Iain S Whitaker ${ }^{1}$

\author{
${ }^{1}$ Reconstructive Surgery \& Regenerative Medicine Research Group, Institute of Life \\ Sciences, Swansea University Medical School, Swansea, UK. \\ ${ }^{2}$ Nuffield Department of Orthopaedics, Rheumatology and Musculoskeletal Sciences, \\ University of Oxford, Botnar Research Centre, Nuffield Orthopaedic Centre, Windmill \\ Road, Oxford, UK \\ ${ }^{3}$ Canniesburn Plastic Surgery Unit, Glasgow Royal Infirmary, North Glasgow University \\ Hospitals NHS Trust, Glasgow, UK
}

Patient reported outcome measures (PROMs) are completed by patients to capture one or more aspects of their health and wellbeing, typically taking the form of standardised and validated questionnaires ${ }^{1}$. They are broadly divided into generic PROMs and site, disease or condition-specific ${ }^{2}$. Their psychometric robustness is of paramount importance, the methods of which and how to assess for this are described in detail in this journal and elsewhere $\mathrm{e}^{2,3}$. The number of PROMs available and their profile amongst clinicians is rising, such as with the recently Royal College of Surgeons advice for the routine collection of PROMs for all those undergoing cosmetic surgery as part of their Cosmetic Surgery Standards ${ }^{5,6}$. It is therefore important that as a speciality we engage with PROMs and identify areas in which PROMs can be most effectively 
harnessed to improve patient care.

The potential applications of PROMs are broader than we think While there is no doubt that asking the patients' perspectives on their condition and treatment is the 'right thing to do', there is also considerable potential to deploy PROMs in innovative ways. PROMs have the potential to generate a positive impact across the patient care journey - informing the patient, clinician and shared decision-making between the two, in addition to affecting the health care system itself from quality assurance to influencing its financial architecture. The breadth of roles for PROMs will be considered in this piece, and ways to overcome barriers and realise these opportunities will be discussed in the accompanying article ("Part 2").

- Informing referrals and surgical decisions

PROMs can be harnessed to improve patient care in the pre-surgical decision-making process. This is already coming into clinical practice in orthopaedics to support decisionmaking around large joint arthroplasty. The Arthroplasty Candidacy Help Engine (ACHE) incorporates outcome measures such as the Oxford Hip and Knee Scores and evidence based thresholds for treatment benefit to predict which patients will actually benefit from arthroplasty ${ }^{8}$. The tool has been deployed to inform shared decision-making, where it is used during the surgical consultation and can inform both the patient and the surgeon of the mathematical likelihood of the patient benefiting from surgery. It could also play a key role in primary care, guiding referral to secondary care, reducing unnecessary referrals, preventing unnecessary surgical intervention for those that are 
likely to benefit least and ultimately improving cost efficiency. Similar systems could be achievable and beneficial in areas of plastic surgery such as Dupuytren's contracture and post-mastectomy breast reconstruction. These conditions both have a similar non-life threatening, but life altering, status to large joint osteoarthritis. We are under pressure to justify treatments that we provide, and many plastic surgery interventions have been labelled with terms like "Procedures of Limited Clinical Effectiveness". Facing the reality of needing to justify value for money spent on plastic surgery, systems like this can support on-going access to treatment for patients who will benefit from them. If there is not resource to fund present or previous numbers of procedures, then rather than stopping that treatment entirely, why not ensure that those who will benefit from it most retain access? This goes beyond "rationing", as such approaches can stop low value and unbeneficial treatments, even if resource were plentiful.

- Optimising and individualising follow-up

Similarly, the post-operative period is open to improved efficiency and better quality from the incorporation of PROMs, through the remote monitoring of recovery and progress in line with expected recovery. At present, follow-up appointments are often made after arbitrary lengths of rehabilitation and may be unnecessary. This is costly to the health care system, to broader society (through loss of productivity and earnings) and to the patient.

Furthermore, monitoring PROMs for general wellbeing and psychological health could be important in identifying those who are struggling emotionally with their surgery and 
health state. This approach has been introduced for children and their parents with chronic illnesses in the Netherlands. Through the KLIK (Kwaliteit van Leven In Kaart Quality of Life in Clinical Practice) programme, PROMs are routinely collected via a web application and allow early identification of problems ${ }^{9}$. Over 10,000 children and parents use this system for diverse conditions, ranging from inflammatory bowel disease to cleft lip and palate. This approach is applicable to much of plastic surgery practice, especially in areas with long-term follow up, like congenital hand surgery or hidradenitis.

- Breathing life into big data

The importance of big data analytics in plastic surgery should not be overlooked in the application of PROMs. If routine PROMs data is collected via digital means a large dataset will soon develop. Linkage of this data with their general electronic health records will open up an exciting new area for data analysis. The potential for discovering new associations and monitoring treatment outcomes on an individual, departmental, national and international level is great.

- Driving reduction in undesirable variation in care The Atlas of Variation (REF) has mapped variation in clinical care for a range of conditions, and variation has been documented for some aspects of plastic surgery too (cite: Davis JHS(E) 2012 vol. 38, 1: pp. 83-85). Routine entry of procedures into registries may help to document and describe variation, and this can be supported by using PROMs. Two of the most relevant registries for plastic surgery are the UK Hand Registry and the UK Flap Registry, and both involve routine collection of PROMs. In 
order to gain the greatest benefit from routine PROMs data collection, standardisation and consensus on the most appropriate outcome measures must be sought. Core Outcome Sets (COS) are agreed upon minimum and standardised sets of outcomes that should be reported on for studies of a specific condition ${ }^{14}$.

- Changing perspectives in life and death scenarios

PROMs are, however, not the panacea for every aspect of healthcare management and decision-making. Their role in life and death decision-making, such as with a large burn, is likely to be limited. However, PROMs data collected on survivors of massive burns could help inform discussions with family members during the early stages of patient management.

- $\quad$ Stimulating interest and driving research agendas

Data arising from routine collection of PROMs can be used to answer existing research questions and may also inspire further questions. The development and refinement of PROMs themselves is also a research-intensive and necessary exercise. For example, one problem with PROMs currently is the degree of responder burden associated with many of them(ref HH paper). This is off-putting to patients and clinicians alike. This is an area of weakness that must be addressed through developments in outcome measurement science. These include the use of item response theory to validate outcome measures, and to ensure that measurement is interval-scaled. Some of the details of this have been discussed recently ${ }^{2,3}$. Related to this, computerised adaptive testing (CAT) is a technique being used to reduce responder burden by selectively delivering questions based on the 
previous responses, so that only questions that will gain more information are used. In practice, this is akin to a situation where someone responds that their hand function is 'normal', then there is little to be gained from asking them increasingly granular questions about hand problems, which may be more appropriate to someone who has 'non-normal' hand function. This will be further improved through the incorporation of routine digital collection (moving away from paper based questionnaires), the use of artificial intelligence and potentially voice recognition software to avoid the need for manual form filling.

Who should be responsible for driving change in PROM use in the plastic surgery community? We believe that we all have a responsibility to engage with and encourage the use of PROMs for our patients. Not only because of the many exciting benefits as outlined above, but also because our patients are asking for it. The enthusiasm for further research into the development and performance of PROMs has been clearly identified as a major research priority in the recent James Lind Alliance exercise for hand surgery ${ }^{7}$. Furthermore, as clinicians we should use PROMs to advocate for treatments that show significant psychological and global quality of life gains, but that and are low on a funder's priority list. Examples of this include massive weight loss surgery and autologous breast reconstruction, where PROMs have demonstrated these procedures as delivering positive gains for patients ${ }^{10,11}$. National bodies such as the Royal College of Surgeons and BAPRAS, along with the Department of Health, should also drive forward the increased use of PROMs. Their increased use will not only lead to better patient care, but in a world of value-based healthcare (ref), will help provide the best outcomes for 
patients as the most cost effective price. PROM data is already used in health technology assessment, where the cost effectiveness of treatments is measured by calculating the incremental cost effectiveness ratio, which is the additional cost incurred by a new treatment, relative to the gain achieved. This gain achieved is often measured in qualityadjusted life years (QALYs), which typically combines overall survival with health status or the utility ${ }^{12}$. These latter elements are calculated using PROM data. It has also been suggested that future health reimbursement systems could be based on PROM data ${ }^{13}$, something that funders in the United States of America are currently investigating (ref).

PROMs can play an important role in clinical decision-making, measuring the effectiveness of interventions and improving the treatments we offer. In order for the plastic surgery community and our patients to truly benefit from this revolution in healthcare we must identify areas of greatest benefit and novel applications for their use.

Being able to accurately measure relevant constructs is particularly valuable in plastic surgery, where the objective of treatment may range across improving hand function, identifying satisfaction with breast reconstruction, or establishing quality of life changes from surgery. This matters in research applications such as clinical trials, and for quality assurance of surgery through routine data collection in clinical practice. The technical aspects of refining measurement to ensure that it is accurate and the process for selecting outcome measures have both been described in recent articles in this journal., ${ }^{1,2}$ Furthermore, the potential breadth of the practical applications of outcome measurement has been discussed in the accompanying editorial ("Part 1"). If such improved 
measurement is expected to benefit patients and clinicians, we must establish how optimised measurement can gain traction in health systems.

There are some mechanisms involving outcome measurement that are mandatory. While mandatory data collection might be construed as undesirable by some clinicians, it also has potential advantages. These advantages may apply at the microsystem level of the individual surgeon or department, or across the health service in general. In contrast, when there is no mandate to drive routine outcome measurement, generating buy-in to data collection is key.

There are examples in the United Kingdom National Health Service (NHS) where outcome measurement has been mandated. With the aim of determining the effectiveness of patient care and service provision, NHS England has established the collection of patient-reported outcome measures (PROMs) following hip and knee replacement, varicose vein surgery and groin hernia repair through the National PROMs Programme. ${ }^{3}$ PROM data for arthroplasty also contributes to The National Joint Registry (NJR). The NJR collects surgical, functional and patientreported outcomes to determine the effectiveness of interventions and improve national clinical standards for hip and knee replacement. ${ }^{4}$ At surgeon or individual centre level, the NJR permits reflective practice and enables the impact of changes to care pathways to be studied. Data can also be used to support revalidation and other quality assurance processes with minimal additional burden on the surgeon at the time of accessing data. The fact that this data collection is mandatory probably explains much of the disparity in completion rate between hip and knee PROMs (mandatory) and shoulder PROMs (not mandatory) in the NJR. ${ }^{5}$ As participation is compulsory, resources are allocated to data 
collection and processing; in the absence of this, individuals might have to collect data without financial or administrative support. Mandatory outcome measurement may expand in plastic surgery too. The Royal College of Surgeons of England has proposed compulsory collection of PROM data for cosmetic surgery. ${ }^{6}$ It is also conceivable that the use of existing registries in plastic surgery may also become compulsory. In the UK, our specialty already has a UK National Hand Registry and a UK National Flap Registry provided by the British Society for Surgery of the Hand and the British Association of Plastic, Reconstructive and Aesthetic Surgeons respectively. ${ }^{7,8}$ If such systems adopt the quality outcome measures and capture a large proportion of the treatment delivered in a system, then formal recognition of their role may follow.

There are other aspects of health service policy that could also act as levers to support effective measurement in clinical practice. These include the NHS Outcomes Framework (NOF), the Commissioning for Quality and Innovation (CQUIN) national goals, and the National Institute for Health and Care Excellence (NICE) Quality Standards. NOF comprises domains spanning clinical effectiveness, patient experience and safety through which local commissioners should commission on the basis of improved outcomes. It is one process for holding NHS Commissioning Board accountable for the health of the nation. ${ }^{9}$ CQUIN mandates quality improvement through incentivising demonstrated improvements in quality and innovation in specified areas of patient care. ${ }^{10}$ Quality Standards represent evidence based markers of cost-effective care for defined conditions. However, currently there is a limited number of outcome measures that can be used as measures of clinical quality. ${ }^{11}$ While such 
processes may incentivise or drive data collection, they have to cover all areas of health care, and plastic surgery may not be prioritised in them.

Where there is no formal mandate to measure outcomes routinely, generating buy-in by stakeholders (including clinicians, patients, and others) is essential if participation and completion is to be high. Some steps towards achieving buy-in include demonstrating the importance and value of data, standardising the use of the best-available outcome measures, ensuring that high quality data are translated into practice through clinical guidelines, and reducing the burden of data collection.

Priority setting partnerships (PSPs) bring together surgeons and other clinicians, patients and healthcare providers to identify and prioritise uncertainties. They may be run by organisations like The James Lind Alliance. ${ }^{12}$ Research priorities that arise from PSPs are validated as being of importance to all represented stakeholders. As well as drawing the attention of major research funders like the National Institute for Health Research (NIHR), they should reiterate the importance of collecting outcome data in these areas in research and potentially in routine practice too. PSPs have been developed for some plastic surgery subspecialties like common hand conditions, cleft lip and palate, and hidradenitis suppurativa. ${ }^{13}$ Other areas of plastic surgery may also benefit if further PSPs are conducted.

If the importance of an area of uncertainty has been highlighted by a PSP, then research into the question should employ the best outcome measurement tools available. Assessing the quality of outcome measures like PROMs can be supported by using existing criteria, such as those provided by the Consensus- 
based Standards for the selection of health status Measurement Instruments (COSMIN) initiative. ${ }^{14}$ Such methods of outcome measurement validation have been discussed and summarised recently. ${ }^{1}$

Even if guidance is followed, there is still likely to be variation in measurement, and this is often undesirable. ${ }^{15}$ Standardisation of outcome measurement could reduce variation, particularly when routine data collection across one or more countries is to be implemented. Core outcome sets (COS) provide a recommended minimum dataset that should be measured and reported when studying a specific condition. ${ }^{16}$ There can be variation in the methods used to generate cOS. The International Consortium for Health Outcomes Measurement (ICHOM) is one organisation working with stakeholders to develop and implement globallyrelevant standard sets of outcome measurements, each specific to a medical condition. ${ }^{17}$ Recognition of the need for COS has stimulated the development of COMET (Core Outcome Measures in Effectiveness Trials) Initiative, ${ }^{18}$ which facilitates the development and dissemination of COS. Outcome measurement is an evolving field, and COS may need to be revised as PROMs with better methodological quality are developed.

Once outcome measurement has been refined and standardised, the burden of data collection can be reduced and waste can be avoided to make routine outcome measurement more achievable. Simplifying data collection and then routinely collecting data in our registries may help. The burden of data collection can also be reduced by techniques such as computerised adaptive testing (CAT). A CAT involves using a computer to administer an appropriate PROM, one question at a time. Using an algorithm, the CAT chooses the subsequent question 
based on the previous answer given. By selecting only the questions that will provide more information, the person's score can be determined without having to ask all of the questions. CATs and standard electronic PROMs could be administered by virtual assistants (such as Siri or Alexa, or similar) to further reduce the manpower required for data collection. If the burden of data collection is reduced through steps like these, then routinely collecting clinical practice data in registries is likely to be more achievable, even when it is not mandatory.

Sharing the load of designing and conducting research through multi-centre collaboration is also beneficial. This may be through project-specific arrangements, or through established collaboratives like The Reconstructive Surgery Trials Network (RSTN).

If efforts are made to collect high quality routine data, in either research studies or in routine clinical practice, then incorporating this evidence into guidelines may create greater impact from these endeavours. Guidelines that meet the standards set in tools like Appraisal of Guidelines for Research and Evaluation (AGREE) II, ${ }^{19}$ may align with health service policy levers, leading to better implementation of their recommendations. For example, in the UK, AGREE II forms the basis for Accreditation of guideline developers by the National Institute for Health and Care Excellence (NICE), with Accredited guidelines able to inform NICE's Quality Standards, which have been discussed above.

These are some of the potential pathways through which outcome measurement could be improved, facilitated and translated into service improvement. Some of these options may not be able to be harnessed. Furthermore, the exact processes 
and policies concerned will vary between countries and between health services, and may change over time. However, fostering a culture of high-quality measurement in plastic surgery, encouraging enthusiasm for data collection in everyday clinical practice as well as in research, and being prepared to embrace innovations in measurement science will all contribute to better understanding of the reality of the conditions we treat, and the true impacts of the interventions that we offer.

Funding statement: JR is funded by the National Institute for Health Research (NIHR). This article presents independent research funded by the NIHR. The views expressed are those of the authors and not necessarily those of the NHS, the NIHR or the Department of Health. TD is funded by the Welsh Clinical Academic Training (WCAT) Fellowship.

\section{References}

1. Devlin, N. J. \& Appleby, J. Getting the most out of PROMS. Putting health outcomes at the heart of NHS decision making. The King's Fund (2010).

2. Wormald, J. C. R. \& Rodrigues, J. N. Outcome measurement in plastic surgery. J Plast Reconstr Aesthet Surg 71, 283-289 (2018).

3. Dobbs, T., Hughes, S., Mowbray, N., Hutchings, H. A. \& Whitaker, I. S. How to decide which patient-reported outcome measure to use? A practical guide for plastic surgeons. Journal of Plastic, Reconstructive \& Aesthetic Surgery (2018). 
doi:10.1016/j.bjps.2018.03.007

4. Lansley, A. Equity and excellence: liberating the NHS. (Department of Health, 2010).

5. Surgeons, R. C. O. Use of Patient Reported Outcome Measures within clinical practice. $1-10$ (2015).

6. Surgeons, R. C. O. Professional Standards for Cosmetic Surgery. (2016).

7. Common Conditions Affecting the Hand and Wrist. BSSH Available at: http://www.bssh.ac.uk/patients/bssh_james_lind_alliance_partnership.aspx (Accessed: 2nd April 2018)

8. Introducing Standardized and Evidence Based Thresholds for Hip and Knee Replacement Surgery -The Arthroplasty Candidacy Help Engine (The ACHE tool). National Institute for Health Research Available at: https://www.journalslibrary.nihr.ac.uk/programmes/hta/116301\#/ (Accessed: 2nd April 2018)

9. Kwaliteit van Leven In Kaart. Kwaliteit van Leven In Kaart Available at: https://www.hetklikt.nu/over.php (Accessed: 2nd April 2018)

10. Jeevan, R. et al. Findings of a national comparative audit of mastectomy and breast reconstruction surgery in England. J Plast Reconstr Aesthet Surg 67, 1333-1344 (2014).

11. Gilmartin, J., Bath-Hextall, F., Maclean, J., Stanton, W. \& Soldin, M. Quality of life among adults following bariatric and body contouring surgery: a systematic review. JBI Database System Rev Implement Rep 14, 240-270 (2016).

12. National Institute for Health and Care Excellence. Guide to the Methods of 
Technology Appraisal 2013. (National Institute for Health and Care Excellence (NICE), 2013).

13. Darzi, A. Our NHS. Our Future. High Quality Care for All. NHS Next Stage Review Final Report. (2008).

14. Porter, M. E., Larsson, S. \& Lee, T. H. Standardizing Patient Outcomes

Measurement. N. Engl. J. Med. 374, 504-506 (2016). 